\title{
Databases for collisional and radiative processes in small molecules needed for spectroscopy use in astrophysics
}

\author{
D. Jevremović ${ }^{1}$, V.A.Srećković ${ }^{2}$, B.P. Marinković ${ }^{2}$ and V. Vujčić ${ }^{1}$ \\ 1 Astronomical Observatory, Volgina 7, 11060 Belgrade, Serbia (E-mail: \\ darko@aob.rs) \\ 2 Institute of Physics Belgrade, BU, Pregrevica 118, 11080 Zemun, Belgrade, \\ Serbia, (E-mail: bratislav.marinkovic@ipb.ac.rs,vlada@ipb.ac.rs)
}

Received: July 30, 2019; Accepted: December 12, 2019

\begin{abstract}
In this contribution we present a report on two atomic/molecular databases for collisional and radiative processes, BEAMDB and MolD, which are web services at the Serbian Virtual Observatory (SerVO) and nodes within the Virtual Atomic and Molecular Data Center (VAMDC). Within the BEAMDB there are mainly electron scattering cross section data, but having in mind the importance of spectroscopic data obtained by particle collisions, we also provide a number of electron energy loss spectra and threshold electron spectra. Electron loss spectroscopy with its complementarity to the photon absorption data, provides valuable information on the optically forbidden atomic and molecular transitions. The MolD database contains photodissociation cross sections for the individual ro-vibrational states of the diatomic molecular ions as well as corresponding data on molecular species and molecular state characterizations. The data can be used for further applications, e.g., for non-local thermal equilibrium modeling of early universe chemistry, modeling of the solar atmosphere, atmospheres of white dwarfs, etc.
\end{abstract}

Key words: Atomic processes - Planets and satellites: general - Line: profiles - Plasmas- ISM: clouds

\section{Introduction}

Obtaining crucial information on the different environments within our universe has become possible with advances in observational astronomy and computer modeling. For instance, data from new telescopes (e.g. ALMA) that will discover and explore the molecular universe (Saldaño et al., 2018, Zakharenko et al. 2019) require vast amount of atomic and molecular data for interepretation of observed spectra. As a consequence atomic and molecular databases have become essential for developing models and simulations of complex physical processes and for the interpretation of data provided by measurements e.g. in laboratory plasmas and in planetary atmospheres (Marinković et al., 2017b a Srećković et al. 2017a). In order to provide a repository of such large amounts of 
data, the creation of a Virtual Observatory and Virtual Data Centre has been crucial (Dubernet et al., 2010, 2016, Juevremović et al., 2009, Srećković et al. 2017b).

In this contribution we present two atomic/molecular databases for collisional and radiative processes, BEAMDB and MolD, which are web services at the Serbian Virtual Observatory (SerVO) and nodes within the Virtual Atomic and Molecular Data Centre (VAMDC). The following section gives an overview of databases. A technical specification is described in Section 3 (an example for the data model is given in the appendix), while the Section 4 summarizes the material.

\section{Database nodes}

The Belgrade nodes of VAMDC are hosted by SerVO and currently contain two atomic/molecular databases of astrophysical importance, BEAMDB (servo. aob.rs/emol) and MolD (servo.aob.rs/mold). Both databases have been built using the specifications and rules required by the VAMDC project (see Dubernet et al. 2016; Moreau et al. 2018). The Belgrade nodes aim to provide a repository for cross sections and rates for reactions needed for astrophysical plasma modeling.

\subsection{MolD}

MolD as node of VAMDC contains photodissociation cross sections for the individual ro-vibrational states of the diatomic molecular ions, reaction rate coefficients as well as corresponding data on molecular species and molecular state characterisations. At the moment MolD contains data for photodissociation cross-sections of the molecular ions such as $\mathrm{He}_{2}^{+}, \mathrm{H}_{2}^{+}, \mathrm{MgH}^{+}, \mathrm{HeH}^{+}, \mathrm{LiH}^{+}$, $\mathrm{NaH}^{+}$, etc., and the corresponding averaged thermal photodissociation crosssections (for details see e.g. table 1 from Marinković et al. 2017b representing species, states, etc.). The database is hosted at the Belgrade Astronomical Observatory, http://servo.aob.rs (Jevremović et al., 2009). The corresponding data are important for exploring the dense molecular clouds in the interstellar medium (ISM), surfaces of small bodies and satellites in our Solar System, modeling of different stellar atmospheres (see the papers Srećković et al. 2018 Vujčić et al. 2015). Collections of such data sets are as well important for the early Universe chemistry and also for understanding the molecular origins of life (Tielens, 2013, Mason et al., 2014).

Recently, the presence of the $\mathrm{HeH}^{+}$molecular ion was confirmed by Güsten et al. (2019) in the planetary nebula NGC 7027. These observations were made possible due to advances in terahertz spectroscopy and high-altitude observatories (Heyminck et al., 2012). This confirmation of the existence of $\mathrm{HeH}^{+}$in interstellar space requires investigation of this molecular ion, in particular the rates of radiative dissociation and association processes. In Fig. 1 we present 


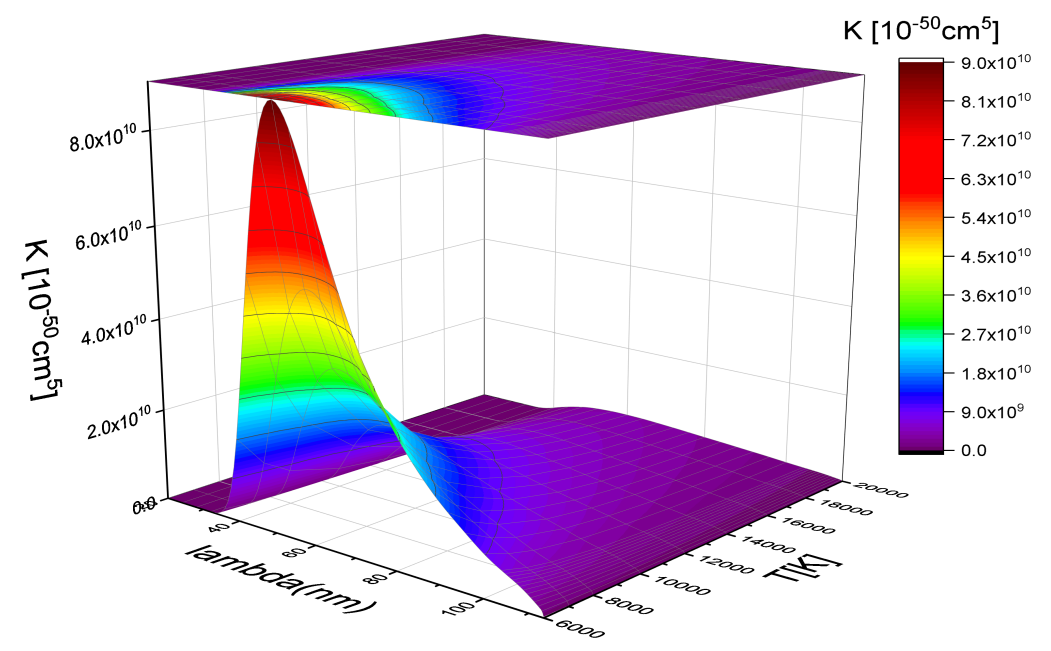

Figure 1. The behaviour of the photodissociation spectral rate coefficients $\mathrm{K}(\mathrm{bf})(\lambda ; T)$ for the molecular ion $\mathrm{HeH}^{+}$.

the behavior of the spectral rate coefficient for photodissociation of the $\mathrm{HeH}^{+}$ molecular ion. For details e.g. partial photodissociation cross sections for the individual ro-vibrational states and molecular state characterisation see MolD. Of course, here we should mention for example the importance of the results connected with the molecular ion $\mathrm{H}_{2}^{+}$(see Figure 4. from Srećković et al. 2017a) and its role in exploring of the interstellar medium, the early Universe chemistry as well as the modeling of different stellar atmospheres (see e.g. Coppola et al. 2013 Babb, 2015, Srećković et al. 2018).

\subsection{BEAMDB}

BEAMDB contains measured collisional data for electron interactions with atoms and molecules in the form of differential and integrated cross sections as well as energy loss of the spectra (Marinković et al. 2015). Two types of electron collision processes are featured in the collection, elastic electron scattering and electron excitation of atoms and electronic states of molecules. The BEAMDB is hosted at the Belgrade Astronomical Observatory, http://servo.aob.rs. One of the aims of BEAMDB is to collect data sets that are related to the electron interaction process which are important for exploring and modeling of different stellar atmospheres and spectroscopy plasma research (Marinković et al. $2017 \mathrm{~b}$ ). It focuses on atomic targets (neutral or ionized), small molecules (diatomic, triatomic, and four-atomic) as well as larger organic and biomolecules. 
a)
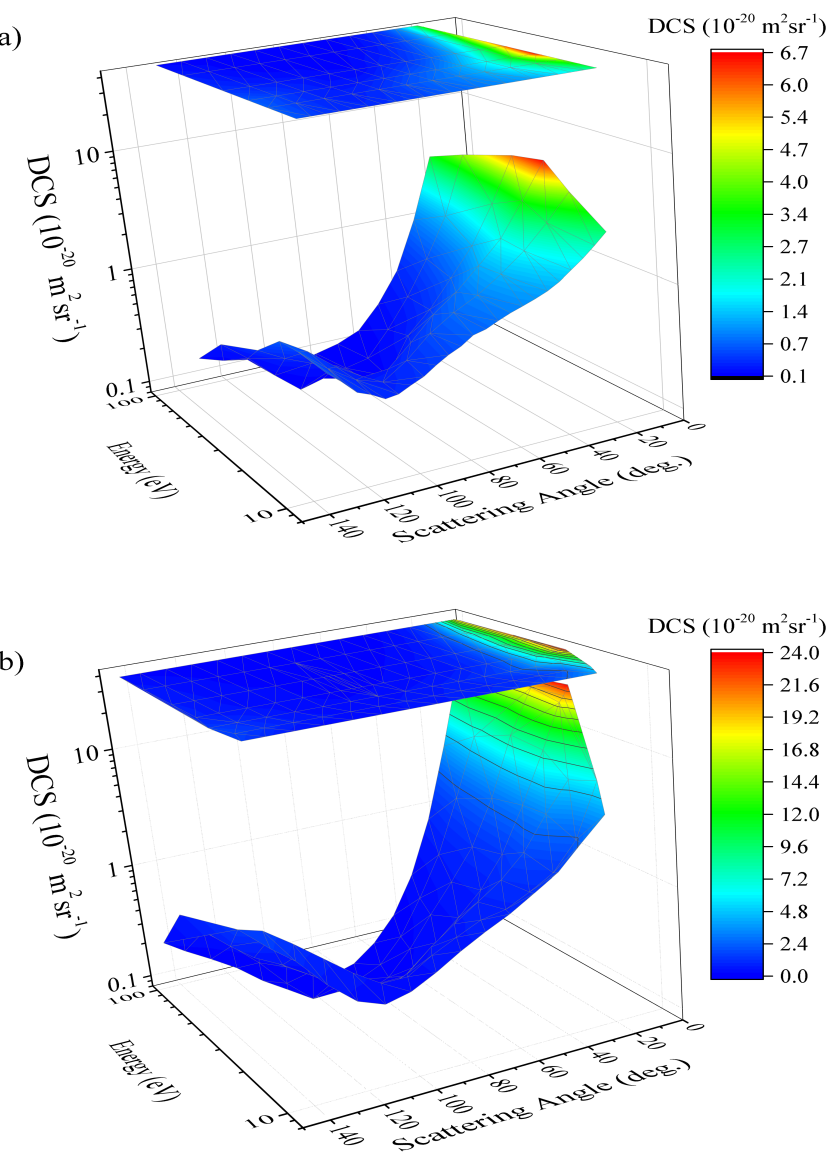

Figure 2. 3-D graph DCS data for electron elastic scattering by nitrous oxide versus electron incident energy and scattering angle. Panel a) data of Marinković and coauthors. panel b) data of Kitajima and coauthors.

Each conference on Spectral Line Shapes in Astrophysics and Plasmas is an opportunity to further develop and enlarge the content of the database with the hot topic atomic/molecular species. Also, special Workshops organized by the scientists in astrophysical and other plasmas contribute to the same aim, just to mention some recent ones: The new view of comet coma processes after Rosetta: The importance of electrons, organized at Comenius University; Bratislava in 2017 (Marinkovic, 2017), Symposium on the Physics of Ionized Gases held in 2018 in Belgrade (Marinković et al. 2018) or Integrations of satellite and ground- 
based observations and multi-disciplinarity in research and prediction of different types of hazards in Solar system in Petnica, Serbia (Marinković et al., 2019).

Comprehensive and up-to-date reviews on electron scattering data have been triennially realised by the IAU Division XII, Commission 14 working group: Atomic collisions (Peach et al. 2009, Peach \& Dimitrijević, 2012, Peach et al. 2015). Data covered by BEAMDB for triatomic molecules include $\mathrm{H}_{2} \mathrm{O}, \mathrm{H}_{2} \mathrm{~S}$ and $\mathrm{N}_{2} \mathrm{O}$. An example of a 3-D graph representing differential cross section (DCS) data for electron elastic scattering by nitrous oxide versus electron incident energy and scattering angle is shown in Fig. 2. Two sets of data are presented from Marinković et al. (1986) (panel a)) and Kitajima et al. (1999) (panel b)) in the energy range from $10 \mathrm{eV}$ to $100 \mathrm{eV}$. One can easily see the forward peaked nature of the elastic cross sections and the variation of magnitude in three orders. Kitajima et al. (2000) have contrasted two sets of data obtained at Sofia University, Tokyo, Japan and at Australian National University, Canberra, Australia, the first ones in the range from $1.5 \mathrm{eV}$ to $100 \mathrm{eV}$ and the second ones from 2 $\mathrm{eV}$ to $20 \mathrm{eV}$. The agreement among these two sets and older measurements is satisfactory having in mind the claimed experimental uncertainties.

\section{Node maintenance}

The most recent module of the VAMDC infrastructure was Query Store by Zwölf et al. (2016) - a mechanism that allows dynamic dataset citation. Adapting the recommendations from the Research Data Alliance (https://www.rdalliance.org/groups/data-citation-wg.html), Query Store grapples with the online datasets citation challenges, from ever-evolving data sources. It should enhance visibility of data providers and reproducability of data sets. Each data response is persisted and versioned so it can be recreated (Moreau et al. 2018) e.g. for the purpose of scientific verification. Furthermore, Query Store can communicate with other scientific services, such as registering a query to Zenodo and obtaining a DOI, see Zwölf et al. (2019).

Technically, Query Store required implementation of a new software layer in the existing NodeSoftware application and a set of asynchronous web services which log data requests (Zwölf et al. 2019). On the side of the Belgrade server, updates of the NodeSoftware Django Web framework were necessary along with minor code alterations.

This contribution presents the continuation of the work performed on database development at Serbian Virtual Observatory. The SerVO is now addressing the challenge of upgrading software and continuous improvements of data processing. New data for targets like triatomic molecules as mentioned above have been constantly added in databases. Although at this moment we concentrate on the intermediate electron energy range from $10 \mathrm{eV}$ to $100 \mathrm{eV}$ (presented in Fig. 2), this range should be enlarged to $1 \mathrm{eV}$ and $1000 \mathrm{eV}$, while there is an increasing interest towards very low energies, down to below $10 \mathrm{meV}$ 
(Kitajima et al., 2019) or to very high energies, up to $100 \mathrm{keV}$ (García, 2019). By decreasing experimental uncertainties and increasing accuracies of theoretical methods, cross section data for rare gas atoms do serve as reference data with the well-established cross sections that may be used as test data in further endeavors.

On the MolD website (http://servo.aob.rs/mold), users can also calculate individual average thermal cross sections or plot them across the available spectrum. The frament of code used for plotting is presented below. The code was shortened for concision. Also, in the appendix we show a sample XSAMS output of a typical query.

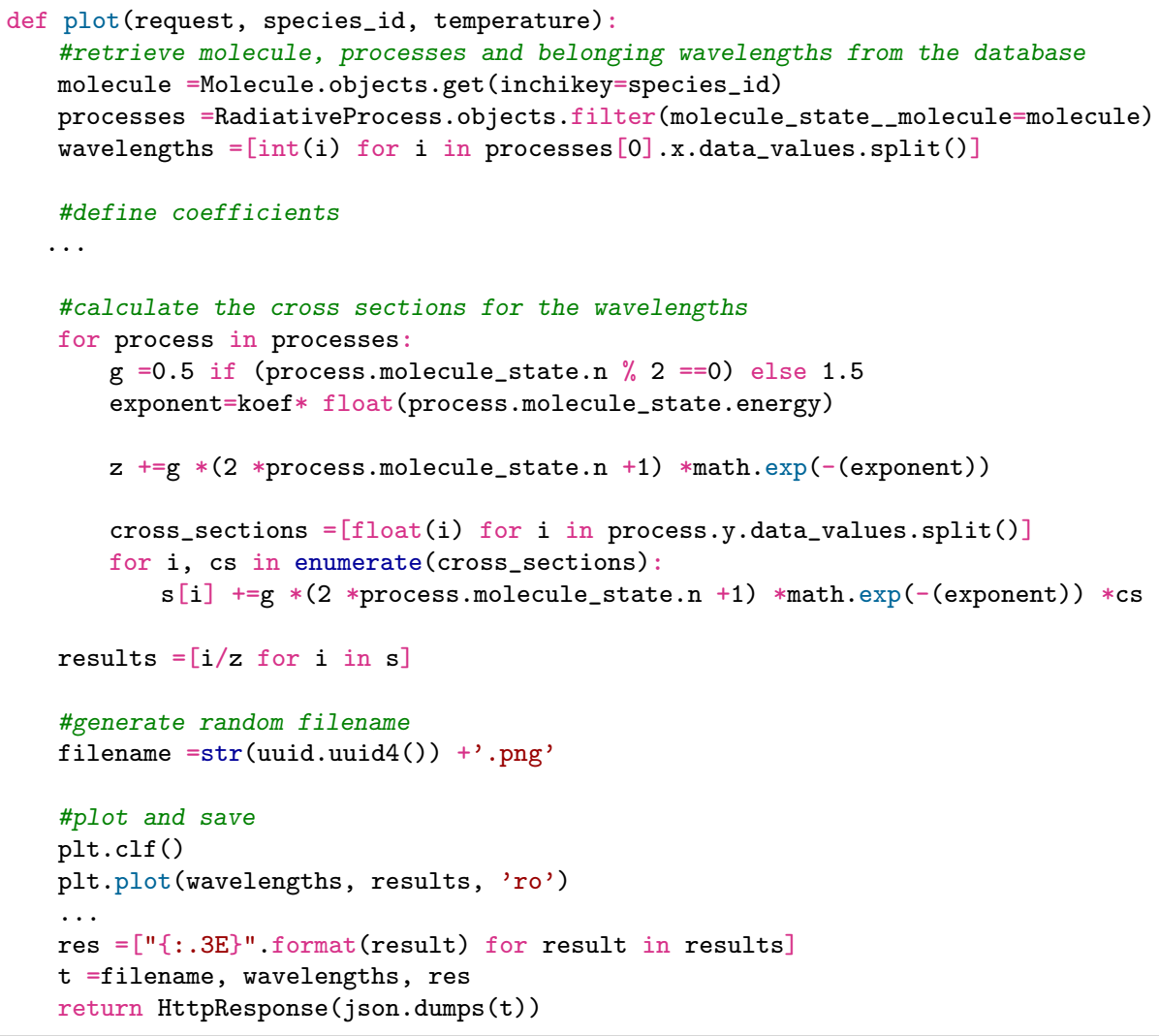

\section{Future developments and concluding remarks}

On the basis of the above, it can be concluded that analysed collisional and radiative atomic/molecular processes represent an important factor in the investigation of a lot of astrophysical environments and features of the interacting 
radiation are important in their spectral analyses. The continuation of developments and services such as atomic/molecular databases and astronomical Virtual Observatories, is crucial in the field of astrophysics and modern science due to its rapid development and makes a huge impact on the way science is done in the developing world.

Acknowledgements. This work is made within projects of the Ministry of Education, Science and Technological Development of Serbia, grant numbers OI176002, III44002. Part of this work has been supported by the VAMDC and the SUP@VAMDC projects funded under the Combination of Collaborative Projects and Coordination and Support Actions Funding Scheme of The Seventh Framework Program. BPM recognizes support from MESTD-RS project OI 171020

\section{References}

Babb, J. F., State Resolved Data for Radiative Association of $\mathrm{H}$ and $\mathrm{H}^{+}$and for Photodissociation of $\mathrm{H}_{2}^{+}$. 2015, Astrophys. J. Suppl. Ser., 216, 21

Coppola, C. M., Galli, D., Palla, F., Longo, S., \& Chluba, J., Non-thermal photons and $\mathrm{H}_{2}$ formation in the early Universe. 2013, Mon. Notices Royal Astron. Soc., 434, 114

Dubernet, M. L., Antony, B. K., Ba, Y. A., et al., The virtual atomic and molecular data centre (VAMDC) consortium. 2016, J. Phys. B, 49, 074003

Dubernet, M. L., Boudon, V., Culhane, J. L., et al., Virtual atomic and molecular data centre. 2010, J. Quant. Spectrosc. Radiat. Transf., 111, 2151

García, G., Invited Talk JS2. 2019, in Proc. XX International Workshop on LowEnergy Positron and Positronium Physics, XXI International Symposium on Electron-Molecule Collisions and Swarms and V Workshop on Non-Equilibrium Processes POSMOL2019, 18 - 20 July 2019 Belgrade, Serbia, Book of Abstracts, ed. D. Cassidy \& et al., (Serbian Academy of Sciences and Arts and Institute of Physics Belgrade, Serbia, 2019), 7

Güsten, R., Wiesemeyer, H., Neufeld, D., et al., Astrophysical detection of the helium hydride ion $\mathrm{HeH}^{+} .2019$, Nature, 568, 357

Heyminck, S., Graf, U., Güsten, R., et al., GREAT: the SOFIA high-frequency heterodyne instrument. 2012, Astron. Astrophys., 542, L1

Jevremović, D., Dimitrijević, M. S., Popović, L. Č., et al., The project of Serbian Virtual Observatory and data for stellar atmosphere modeling. 2009, New Astron. Rev., 53, 222

Kitajima, M., Ejiri, T., Okumura, T., et al., Topical Lecture EMS 15. 2019, in Proc. XX International Workshop on Low-Energy Positron and Positronium Physics, XXI International Symposium on Electron-Molecule Collisions and Swarms and $V$ Workshop on Non-Equilibrium Processes POSMOL2019 18 - 20 July 2019 Belgrade, Serbia, Book of Abstracts,, ed. D. Cassidy \& et al., (Serbian Academy of Sciences and Arts and Institute of Physics Belgrade, Serbia, 2019), 40 
Kitajima, M., Sakamoto, Y., Gulley, R., et al., Electron scattering from N2O: absolute elastic scattering and vibrational excitation. 2000, J. Phys. B, 33, 1687

Kitajima, M., Sakamoto, Y., Watanabe, S., et al., Absolute elastic differential crosssections for electron scattering by N2O at 1.5-100 eV. 1999, Chem. Phys. Lett., 309, 414

Marinković, B. P., Electron Impact Excitation data for $\mathrm{H}_{2} \mathrm{O}, \mathrm{N}_{2} \mathrm{O}$ and $\mathrm{H}_{2} \mathrm{~S}$ triatomic molecules. 2017, in Book of Abstracts, Workshop: The new view of comet coma processes after Rosetta: The importance of electrons, Comenius University; Bratislava, Slovakia, May 24 to 26 2017, ed. N. Mason, J. Országh, P. Papp, \& Š. Matejčík, The Workshop was organized under the ELEvaTE grant agreement number 692335, $10-11$

Marinković, B. P., Bredehöft, J., Vujčić, V., Jevremović, D., \& Mason, N., Rosetta Mission: Electron Scattering Cross Sections-Data Needs and Coverage in BEAMDB Database. 2017a, Atoms, 5, 46

Marinković, B. P., Ivanović, S., Uskoković, N., \& Nešić, M., Electron-impact cross sections for tholins: Coverage within BEAMDB databases. 2019, in Proc. Integrations of satellite and ground-based observations and multi-disciplinarity in research and prediction of different types of hazards in Solar system, ed. A. Nina, M. Radovanović, \& V. A. Srećković, 10 - 13 May 2019, Petnica Science Center, Valjevo, Serbia, Book of Abstracts (Europlanet 2020 RI NA1 Innovation through Science Networking and Geographical Institute 'Jovan Cvijić' of Serbian Academy of Sciences and Arts, Belgrade, 2019), Abstract of Posters, 47-48

Marinković, B. P., Jevremović, D., Srećković, V. A., et al., BEAMDB and MolD databases for atomic and molecular collisional and radiative processes: Belgrade nodes of VAMDC. 2017b, Eur. Phys. J. D, 71, 158

Marinković, B. P., Srećković, V. A., Jevremović, D., et al., BEAMDB and MolD Collisional and Radiative Databases at the Serbian Virtual Observatory. 2018, in Proc. 29th Summer School and Int. Symp. on the Physics of Ionized Gases (SPIG2018), Contributed Papers $\& 5$ Abstracts of Invited Lectures, Topical Invited Lectures, Progress Reports and Workshop Lectures, ed. G. Poparić, B. Obradović, D. Borka, \& M. Rajković, (Vinča Institute of Nuclear Sciences and Serbian Academy of Sciences and Arts, Belgrade, 2018). Contributed Paper, 23-26

Marinković, B. P., Szmytkowski, C., Pejčev, V., Filipović, D., \& Vušković, L., Differential cross sections for elastic and inelastic scattering of electrons by $\mathrm{N} 2 \mathrm{O}$ in the range from 10 to $80 \mathrm{eV}$. 1986, J. Phys. B, 19, 2365

Marinković, B. P., Vujčić, V., Sushko, G., et al., Development of collisional data base for elementary processes of electron scattering by atoms and molecules. 2015, Nucl. Instrum. Methods Phys. Res. B, 354, 90

Mason, N. J., Nair, B., Jheeta, S., \& Szymańska, E., Electron induced chemistry: a new frontier in astrochemistry. 2014, Faraday discussions, 168, 235

Moreau, N., Zwolf, C.-M., Ba, Y.-A., et al., The VAMDC Portal as a Major Enabler of Atomic and Molecular Data Citation. 2018, Galaxies, 6, 105 
Peach, G. \& Dimitrijević, M. S., Division XII / Commission 14 / Working Group Collision Processes. 2012, Transactions of the International Astronomical Union, Series A, 28, 371

Peach, G., Dimitrijević, M. S., \& Barklem, P. S., Division B Commission 14 Working Group: Collision Processes. 2015, Transactions of the International Astronomical Union, Series A, 29, 120

Peach, G., Dimitrijević, M. S., \& Stancil, P. C., Division XII / Commission 14 / Working Group Collision Processes. 2009, Transactions of the International Astronomical Union, Series A, 27, 385

Saldaño, H. P., Rubio, M., Jameson, K., \& Bolatto, A. D., Molecular cloud properties in low-metallicity star forming regions. 2018, Boletin de la Asociacion Argentina de Astronomia La Plata Argentina, 60, 192

Srećković, V. A., Dimitrijević, M. S., Ignjatović, L. M., Bezuglov, N., \& Klyucharev, A., The Collisional Atomic Processes of Rydberg Hydrogen and Helium Atoms: Astrophysical Relevance. 2018, Galaxies, 6, 72

Srećković, V. A., Ignjatović, L. M., Jevremović, D., Vujčić, V., \& Dimitrijević, M. S., Radiative and Collisional Molecular Data and Virtual Laboratory Astrophysics. 2017a, Atoms, 5, 31

Srećković, V. A., Jevremović, D., Vujčić, V., et al., Mol-D a Database and a Web Service within the Serbian Virtual Observatory and the Virtual Atomic and Molecular Data Centre. 2017b, in IAU Symposium, Vol. 325, Astroinformatics, ed. M. Brescia, S. G. Djorgovski, E. D. Feigelson, G. Longo, \& S. Cavuoti, 393-396

Tielens, A., The molecular universe. 2013, Reviews of Modern Physics, 85, 1021

Vujčić, V., Jevremović, D., Mihajlov, A. A., et al., MOL-D: A Collisional Database and Web Service within the Virtual Atomic and Molecular Data Center. 2015, J. Astrophys. Astron., 36, 693

Zakharenko, O., Lewen, F., Ilyushin, V. V., et al., Rotational spectroscopy of isotopic species of methyl mercaptan at millimeter and submillimeter wavelengths: CH334SH. 2019, Astron. Astrophys., 627, A41

Zwölf, C. M., Moreau, N., Ba, Y.-A., \& Dubernet, M.-L., Implementing in the VAMDC the New Paradigms for Data Citation from the Research Data Alliance. 2019, Data Science Journal, 18

Zwölf, C. M., Moreau, N., \& Dubernet, M.-L., New model for datasets citation and extraction reproducibility in VAMDC. 2016, J. Mol. Spectrosc., 327, 122

\section{A. Node software}

\section{A.1. XSAMS generated output example}

A sample query for Lithium cations with specific atomic numbers

select * where InchiKey='HSOYNNFNUCWPIZ-UHFFFAOYSA-N' and MoleculeQNJ=1 and MoleculeQNv=5 
This is an annotated example of an XSAMS document returned from a query. The return data are from the MolD database node. The output was shortened for concision.

$<? x m l$ version="1.0" encoding="UTF-8"?>

<XSAMSData xmlns="http://vamdc.org/xml/xsams/1.0" xmlns:xsi="http://www. w3.org/2001/XMLSchema-instance" xmlns:cml="http://www.xml-cml.org/ schema" xsi:schemaLocation="http://vamdc.org/xml/xsams/1.0 http:// vamdc.org/xml/xsams/1.0">

<Sources><Source sourceID="BmolD-2019-07-25-20-5-16">

<Comments>Query was: select * where ( (InchiKey = 'HSOYNNFNUCWPIZUHFFFAOYSA-N')) and MoleculeQNJ=1 and MoleculeQNv=5

Query Store Link: https://querystore.vamdc.eu/GetUUIDByToken?queryToken= mold: 951 f47f7-bdfb-45f0-8939-e7fc9f533a42: get</Comments>

$<$ Year $>2019</$ Year $>$

$<$ Category>database</Category>

<ProductionDate>2019-07-25</ProductionDate>

$</$ Source $>$

$</$ Sources $>$

<Species>

$<$ Molecules>

<Molecule speciesID="XmolD-3">

$<$ MolecularChemicalSpecies>

$<$ OrdinaryStructuralFormula $><$ Value $>$ LiH $+</$ Value $></$ OrdinaryStructuralFormula $><$ StoichiometricFormula>LiH+</StoichiometricFormula>

$<$ ChemicalName $><$ Value $>$ Lithium hydride cation</Value $></$ ChemicalName $>\langle$ InChI $>$ 1S/Li.H/q+1</InChI><InChIKey>HSOYNNFNUCWPIZ-UHFFFAOYSA-N</InChIKey>

$<$ VAMDCSpeciesID></VAMDCSpeciesID>

<StableMolecularProperties>

$</$ StableMolecularProperties $>$

$</$ MolecularChemicalSpecies>

<MolecularState auxillary="true" stateID="SmolD-1259"><Description> ionized</Description> <MolecularStateCharacterisation> </ MolecularStateCharacterisation $>$

<Case xsi:type="case:Case" caseID="dcs" xmlns: case="http://vamdc.org/xml/ xsams/1.0/cases/dcs" $><$ case: QNs $></$ case: QNs $></$ Case $></$ MolecularState $><$ MolecularState auxillary="false" stateID="SmolD-1273" $><$ Description $>r$ : 1, v: 5</Description> <MolecularStateCharacterisation><StateEnergy energyOrigin="SmolD-1259" $><$ Value units="au" $>-.243083268582 E-04$

$</$ Value ></StateEnergy> </MolecularStateCharacterisation>

<Case xsi:type="case:Case" caseID="dcs" xmlns:case="http://vamdc.org/xml/

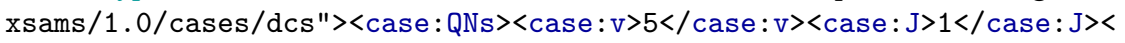
/case: QNs $></$ Case $></$ MolecularState $></$ Molecule $>$

$</$ Molecules $>$

$\langle/$ Species $>$

$\langle$ Processes>

<Radiative>

$<$ AbsorptionCrossSection id="PmolD-CS1328" $><$ SourceRef $>$ BmolD-3 $</$ SourceRef $><$ SourceRef $>$ BmolD-4</SourceRef $><$ SourceRef $>$ BmolD $-5</$ SourceRef $><$ 
Description $></$ Description $><\mathrm{X}$ units $=" \mathrm{~nm} "><$ DataList count='151'>50 51 $525354555657585960 \ldots</$ DataList $\rangle\langle/ \mathrm{X}\rangle\langle\mathrm{Y}$ units $=" \mathrm{~cm} 2 "><$ DataList count='151'>0.714843E-21 $0.732920 \mathrm{E}-21 \quad 0.750881 \mathrm{E}-210.769486$ $\begin{array}{llllllll}\text { E-21 } & 0.787933 \mathrm{E}-21 & 0.808199 \mathrm{E}-21 & 0.827278 \mathrm{E}-21 & 0.847998 \mathrm{E}-21 & 0.867653 \mathrm{E}\end{array}$ $-210.889914 \mathrm{E}-21 \ldots$

$</$ DataList $></$ Y $>\langle$ Species $><$ SpeciesRef $>$ XmolD $-3</$ SpeciesRef $>\langle$ StateRef $>$ SmolD $-1273</$ StateRef $></$ Species $><$ BandAssignment $></$ BandAssignment $></$

AbsorptionCrossSection $></$ Radiative $>$

$</$ Processes $>$

$</$ XSAMSData $>$ 